\title{
Functionalized multiwall carbon nanotubes incorporated polymer/fullerene hybrid photovoltaics
}

\author{
N. A. Nismy, ${ }^{\text {a) }}$ A. A. D. T. Adikaari, and S. R. P. Silva \\ Advanced Technology Institute, University of Surrey, Guildford GU2 7XH, United Kingdom
}

(Received 9 March 2010; accepted 28 June 2010; published online 20 July 2010)

\begin{abstract}
Multiwall carbon nanotubes are introduced into poly(3-hexylthiophene) and $[6,6]$ phenyl $\mathrm{C}_{61}$ butyric acid methyl fullerene, bulk heterojunction organic photovoltaic devices. Utilization of nanotubes requires chemical modifications for compatibility with solution processable photovoltaics. Better dispersions of carbon nanotubes in organic solvents are achieved by acid functionalization of tubes. Pristine and acid treated multiwall carbon nanotubes have been incorporated into the photoactive layer and better results in fill factors of $62 \%$ and efficiency of $2.3 \%$ are achieved under Air Mass 1.5 Global illumination through the use of acid treated nanotubes. (C) 2010 American Institute of Physics. [doi:10.1063/1.3464970]
\end{abstract}

Photovoltaics (PVs) are seen as the key route to a sustainable energy for future which facilitates the harnessing of solar irradiation into electricity. Initial PV structures were based on inorganic materials and during the last three decades there has been significant effort to develop PVs based on organic materials due to their ease of processing, high light absorption coefficient, and potential for low cost thin film device fabrication. ${ }^{1,2}$

Light absorption, exciton dissociation and charge transport to the respective electrodes are the principle mechanisms governing efficient solar energy harvesting in organic PVs (OPVs). The early research on OPVs was based on single layer materials sandwiched between two metal electrodes with different work functions. ${ }^{3}$ Poor exciton dissociation in these single layered structures was addressed by bilayer device architecture. ${ }^{4}$ However, the bilayer devices were affected by the low diffusion lengths of excitons/carriers in organic materials. The discovery of the fast excited charge transfer from the polymer to the fullerene ${ }^{5}$ and blending of these materials for nanoscale random phase separation led to the bulk heterojunction (BHJ) architecture. ${ }^{6}$ The most commonly used OPV material combination, which exploits the BHJ concept is poly(3-hexylthiophene) (P3HT) and [6,6]phenyl $\mathrm{C}_{61}$ butyric acid methyl fullerene (PCBM).

Carbon nanotubes have been incorporated into OPVs to utilize its exceptional charge transport properties and to provide good dissociation centers. ${ }^{7-9}$ During this project we tried to utilize the properties of multiwall carbon nanotubes (MWCNTs) which posses $s p^{2}$ hybridized $\mathrm{C}-\mathrm{C}$ configurations, with extended $\pi$-electron systems of the back bone. ${ }^{10}$ Carbon nanotube dispersion and preparation of a homogeneous solution play a major role in thin film PV device fabrication processes. The inherent difficulty in dispersing nanotubes in organic solvents has hindered its utilization in OPVs. Acid functionalization processes tailor the properties of MWCNTs (Ref. 11) and modifies the fully saturated $s p^{2}$ $\mathrm{C}-\mathrm{C}$ bonding in the nanotube sidewalls. ${ }^{12}$ Simultaneously, this process makes nanotubes shorter and the open ends are terminated by carboxylic acid groups. ${ }^{13}$ The oxidative treatment leads to better dispersions of nanotubes in organic

${ }^{\text {a)} E l e c t r o n i c ~ m a i l: ~ n . n i s m y @ ~ s u r r e y . a c . u k . ~}$ solvents ${ }^{14}$ which then gives rise to better results in organic solar cells. ${ }^{15-17}$

In this work, we performed acid functionalization on MWCNTs and studied the effect of functionalization of MWCNTs in OPV characteristics. Acid functionalized MWCNTs (O-MWCNTs) and pristine MWCNTs have been introduced at $0.33 \mathrm{wt} \%$ and compared the effect of functionalization of nanotubes in OPVs. MWCNTs, P3HT, and PCBM were purchased from Sigma Aldrich, Rieke Metals (USA) and Solenne (The Netherlands), respectively. The MWCNTs were dispersed in a 1:3 mixture of nitric and sulphuric acid and was sonicated for $20 \mathrm{~min}$. The mixture was then heated in an oil bath at $130{ }^{\circ} \mathrm{C}$ for $2 \mathrm{~h}$. The acid treated MWCNTs were washed with de-ionized water and centrifuged at $8500 \mathrm{rpm}$ for $10 \mathrm{~min}$, three times. The centrifuged solution was filtered by a polytetrafluroethylene membrane $(0.2 \mu \mathrm{m})$. For the active layer of OPVs, material blends of $0.1 \mathrm{mg}$ of pristine-MWCNTs and $0.1 \mathrm{mg}$ of O-MWCNTs were dispersed in $1 \mathrm{ml}$ of 1,2 dichlorobenzene (DCB) each and sonicated for $1 \mathrm{~h} .15 \mathrm{mg}$ of P3HT was then added to each of these solutions and stirred for $3 \mathrm{~h}$ and $15 \mathrm{mg}$ of PCBM was added and the solution was stirred overnight. Photovoltaic devices were fabricated on indium tin oxide (ITO) coated glass substrates. The substrates were cleaned using an ultrasonic cleaning process (acetone, methanol) and an oxygen plasma. The blends of P3HT:(pristine, O) MWCNTs:PCBM were deposited onto ITO coated substrates by two step spin coating process. The first step was at 1000 $\mathrm{rpm}$ for $40 \mathrm{~s}$ and second step was at $1500 \mathrm{rpm}$ for $5 \mathrm{~s}$. The spin coated films were allowed for dry slowly in a closed glass Petri dish (60 $\mathrm{mm}$ diameter and $15 \mathrm{~mm}$ height) at room temperature for $40 \mathrm{~min}$ and annealed at $125^{\circ} \mathrm{C}$ for $10 \mathrm{~min}$. This process was carried out in a nitrogen filled MBRAUN glove box. The exciton blocking layer, bathocuproine and the aluminium electrode were thermally evaporated using a shadow mask under vacuum at the rate of $2 \AA / \mathrm{s}$ and $3 \AA / \mathrm{s}$, respectively. The active area of the devices were $76 \mathrm{~mm}^{2}$. Immediately after the device fabrication, current-voltage measurements were collected using a Keithley 2425 source meter exposing the devices to a $300 \mathrm{~W}$ Xe Arc lamp ORIEL simulator, fitted with an Air Mass 1.5 Global (AM 1.5G) filter, calibrated to an intensity of $1000 \mathrm{Wm}^{-2}$. 

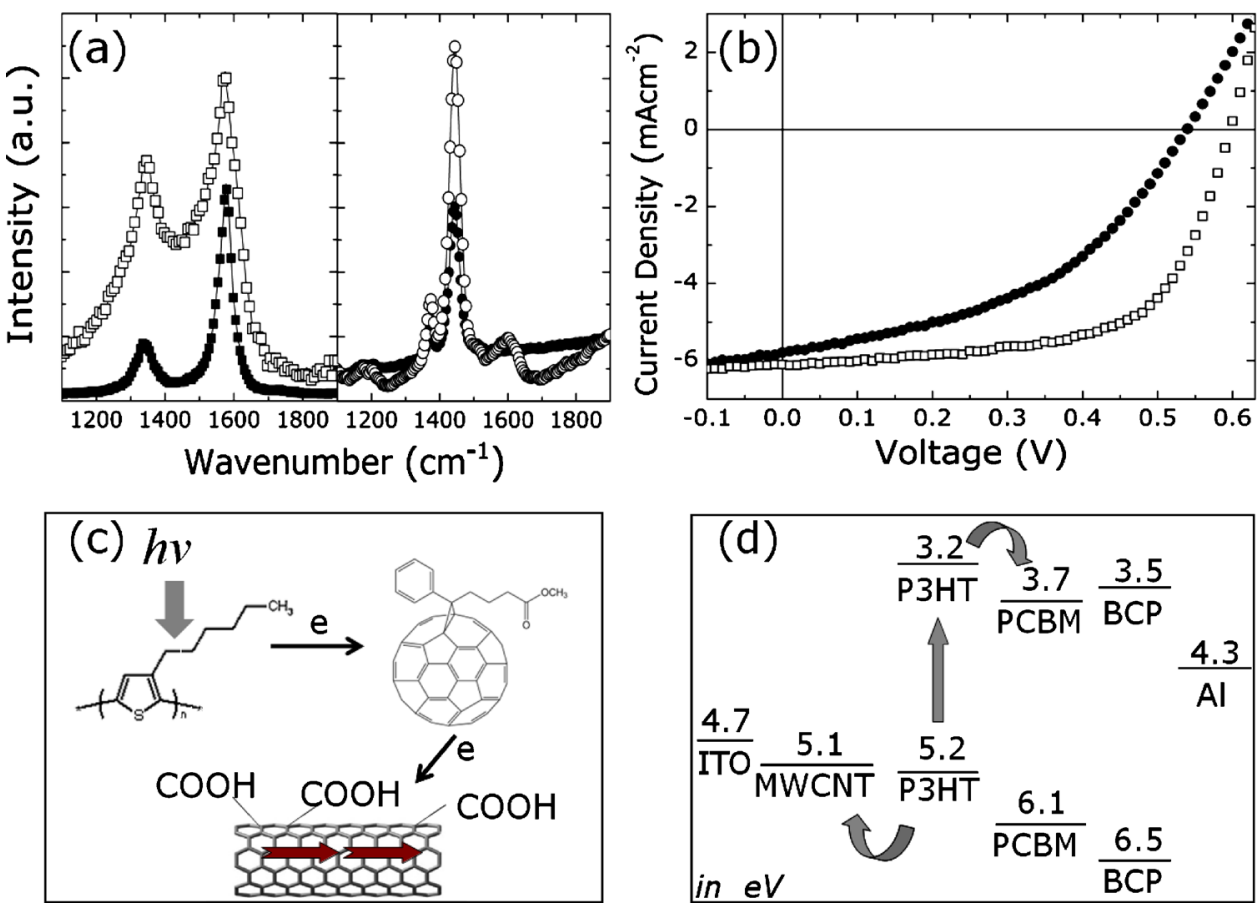

FIG. 1. (Color online) (a) Raman spectra for the pristine MWCNTs (black squares), O-MWCNTs (white squares), P3HT:PCBM films (black circles), and P3HT:OMWCNT:PCBM films (white circles). (b) J-V characteristics of the P3HT:PCBM devices fabricated with 0.33 wt $\%$ of O-MWCNTs (white squares) and of pristine MWCNTs (black circles), (c) schematic diagram of carrier transport in the structure, and (d) suggested energy band alignment diagram for P3HT:OMWCNT:PCBM device.
In order to ascertain the functionalization process, Raman spectroscopy measurements were carried out for the pristine and O-MWCNTs and shown in Fig. 1(a). The ratio of intensities of the $\mathrm{D}$ and $\mathrm{G}$ bands gives information on the structure of the carbon material and an increased ratio is attributed to disorder in the structure. ${ }^{18}$ From the data, the calculated $\mathrm{I}_{\mathrm{D}} / \mathrm{I}_{\mathrm{G}}$ ratio for pristine MWCNTs was $\sim 0.4$ and for O-MWCNTS was $\sim 0.7$, which confirms that upon functionalization, defects are introduced into the nanotube structure and these defects are terminated by carboxylic groups grafting onto the nanotubes. ${ }^{19}$

Figure 1(b) shows current density-voltage (J-V) curves for the devices. J-V curve for the device made with 0.33 wt $\%$ of pristine MWCNT (circles) shows an open circuit voltage $\left(\mathrm{V}_{\mathrm{oc}}\right)$ of $0.54 \mathrm{~V}$, short circuit current density $\left(\mathrm{J}_{\mathrm{sc}}\right)$ of $5.80 \mathrm{~mA} \mathrm{~cm}{ }^{-2}$ and a fill factor $(\mathrm{FF})$ of $44.4 \%$ resulting in maximum power conversion efficiency (PCE) of $1.39 \%$. However, the O-MWCNT incorporated device shows improvements in both $\mathrm{V}_{\mathrm{oc}}(0.60 \mathrm{~V})$ and $\mathrm{J}_{\mathrm{sc}}\left(6.00 \mathrm{~mA} \mathrm{~cm}^{-2}\right)$ along with considerably higher FF $(62.3 \%)$. These devices yielded a maximum PCE of $2.28 \%$. It can be observed that there are improvements in all the fundamental device characteristics upon acid functionalization. The PCE observed for O-MWCNT incorporated devices is one of the highest reported so far, compared to recently published results $2.0 \%{ }^{20}$

We fabricated reference devices, without carbon nanotubes, for comparison purposes. The performance of the reference device is marginally better than the MWCNT incorporated device with a maximum measured efficiency of $2.5 \%$. In the reference device, the total area of the P3HT:PCBM has been exposed to the light, whereas in nanotube incorporated devices some of the area is occupied by the nanotubes and this may affect the absorption.

In order to investigate the nanotube distribution and dispersion in the active layer, scanning electron microscopy images (SEM) of spin coated active layers of P3HT:PCBM [(a) and (b)], P3HT:pristine-MWCNTs:PCBM [(c) and (d)], and P3HT:O-MWCNTs:PCBM [(e) and (f)] are shown in Fig. 2 at lower [(a), (c) and, (e)] and higher [(b), (d), and (f)] magnifications. Bundles of nanotubes can be observed for the untreated MWCNTs incorporated polymer:fullerene blend. However, with the treated nanotubes, a better distribution in the photoactive layer can be observed due to the improved dispersion of O-MWCNTs. Treated MWCNTs have provided a smoother structure with P3HT:PCBM than the pristine MWCNTs which can be associated with the high FF obtained from O-MWCNTs incorporated devices. However, the low concentration of MWCNTs and the embedded dispersion of nanotubes in polymer fullerene hybrid composite, prevents imaging of nanotubes in a typical basis. Furthermore, the existence of nanotubes in the spin coated active layer was confirmed by the Raman spectroscopy [Fig. 1(a)].

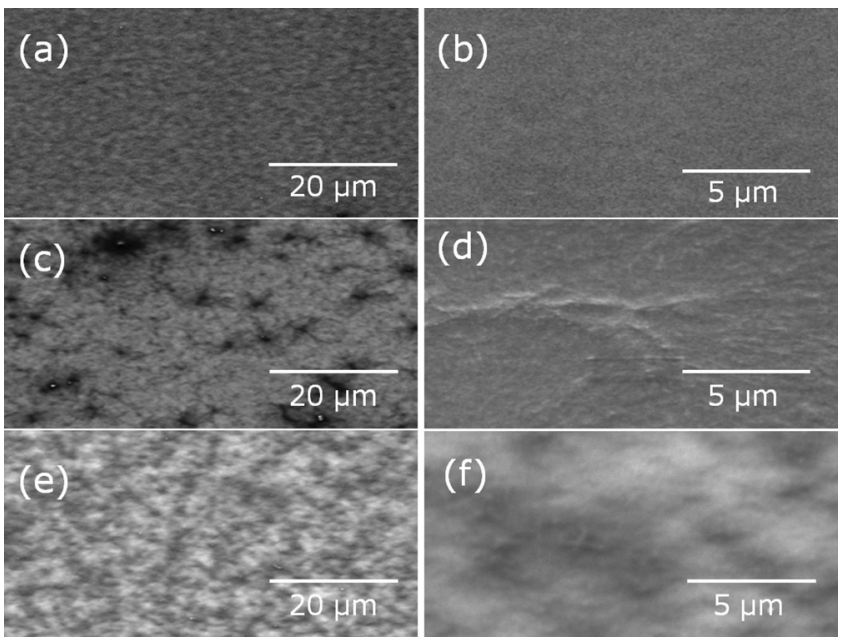

FIG. 2. SEM micrographs of the spin coated P3HT:PCBM [(a) and (b)], P3HT:pristine MWCNTs:PCBM [(c) and (d)], and P3HT:OMWCNTsPCBM [(e) and (f)] active layers at lower and higher magnifications, respectively. 
The role of nanotubes in the devices includes dissociation of excitons where the holes are transported by a hopping mechanism and the electrons transferred through the nanotube as shown in the inset of Fig. 1(c). A possible energy diagram for the charge generation upon photo excitation is shown in Fig. 1(d). The introduction of nanotubes may provide ballistic pathways for the electrons and hence can increase the effective carrier mobility in the active layer. The increased $\mathrm{J}_{\mathrm{sc}}$ values achieved from the O-MWCNTs implies acid treatment of MWCNTs is better for charge transport than the pristine MWCNTs, offering fast exciton dissociation. Furthermore, a percolation network created by the nanotubes is of great importance in transporting charge from one nanotube to another, and affords additional charge transfer pathways. Therefore, it is proposed that the observed enhancements of the OPV device characteristics is due to the acid functionalization process which creates better polymer/ nanotube interfaces in the photoactive layer offering good dissociation centers.

In summary, we have fabricated OPV devices with MWCNTs incorporated into the active layer. The acid functionalization procedure was able to disperse MWCNTs in DCB and allowed us to prepare homogeneous solutions for thin film device fabrication. The $0.9 \%$ increase in PCE and $17 \%$ increase in FF compared to the devices made with pristine MWCNTs imply the suitable addition of functional groups could enhance the device characteristics. Furthermore a PCE of 2.3\% was obtained with the use of O-MWCNTs. Careful selection of the additional functional groups to the nanotubes could make a significant impact in OPVs.

The authors would like to thank Dr. V. Stolojan for useful discussions on electron microscopy. This report is based on a project which is funded by E.ON AG, as part of the
E.ON International Research Initiative. Responsibility for the content of this publication lies with the authors.

${ }^{1}$ P. Peumans, V. Bulovic, and S. R. Forrest, Appl. Phys. Lett. 76, 2650 (2000).

${ }^{2}$ A. A. D. T. Adikaari, D. M. N. M. Dissanayake, and S. R. P. Silva, IEEE J. Sel. Top. Quantum Electron. (to be published), DOI: 10.1109/ JSTQE.2010.2040464.

${ }^{3}$ H. Kallmann and M. Pope, J. Chem. Phys. 30, 585 (1959).

${ }^{4}$ C. W. Tang, Appl. Phys. Lett. 48, 183 (1986).

${ }^{5}$ N. S. Sariciftci, L. Smilowitz, A. J. Heeger, and F. Wudl, Science 258, 1474 (1992).

${ }^{6}$ G. Yu, J. Gao, J. C. Hummelen, F. Wudl, and A. J. Heeger, Science 270, 1789 (1995).

${ }^{7}$ E. Kymakis and G. A. J. Amaratunga, Appl. Phys. Lett. 80, 112 (2002).

${ }^{8}$ E. Kymakis, I. Alexandrou, and G. A. J. Amaratunga, J. Appl. Phys. 93, 1764 (2003).

${ }^{9}$ B. Pradhan, S. K. Batabyal, and A. J. Pal, Appl. Phys. Lett. 88, 093106 (2006).

${ }^{10}$ J. N. Coleman, S. Curran, A. B. Dalton, A. P. Davey, B. McCarthy, W. Blau, and R. C. Barklie, Phys. Rev. B 58, R7492 (1998).

${ }^{11}$ J. Chen, M. A. Hamon, H. Hu, Y. Chen, A. M. Rao, P. C. Eklund, and R. C. Haddon, Science 282, 95 (1998).

${ }^{12}$ E. Cho, H. Kim, C. Kim, and S. Han, Chem. Phys. Lett. 419, 134 (2006)

${ }^{13}$ P. W. Chiu, G. S. Duesberg, U. Dettlaff-Weglikowska, and S. Roth, Appl. Phys. Lett. 80, 3811 (2002).

${ }^{14}$ B. I. Kharisov, O. V. Kharissova, H. Leija Gutierrez, and U. O. Méndez, Ind. Eng. Chem. Res. 48, 572 (2009).

${ }^{15}$ R. A. Hatton, N. P. Blanchard, L. W. Tan, G. Latini, F. Cacialli, and S. R. P. Silva, Org. Electron. 10, 388 (2009).

${ }^{16}$ R. A. Hatton, A. J. Miller, and S. R. P. Silva, J. Mater. Chem. 18, 1183 (2008).

${ }^{17}$ A. J. Miller, R. A. Hatton, and S. R. P. Silva, Appl. Phys. Lett. 89, 133117 (2006).

${ }^{18}$ M. S. Dresselhaus, G. Dresselhaus, R. Saito, and A. Jorio, Phys. Rep. 409, 47 (2005).

${ }^{19}$ S. Osswald, M. Havel, and Y. Gogotsi, J. Raman Spectrosc. 38, 728 (2007).

${ }^{20}$ S. Berson, R. de Bettignies, S. Bailly, S. Guillerez, and B. Jousselme, Adv. Funct. Mater. 17, 3363 (2007). 